\title{
Conceptualizaciones acerca de los animales en niños de la sociedad mayoritaria y de la comunidad indígena Uitoto en Colombia
}

\author{
Conceptualizations about animals in children from \\ the majority society and Uitoto Colombian native \\ community
}

\author{
Pablo Páramo \\ Carlos Julio Galvis ${ }^{1}$
}

\section{Resumen}

El estudio se orientó a identificar los vínculos que desarrollan niños de diferentes contextos culturales y regionales de Colombia (de la sociedad mayoritaria e indígena) sobre los animales a través de sus constructos personales. Las clasificaciones realizadas por los niños de la sociedad mayoritaria e indígenas siguieron un sistema centrado en constructos ecológicos-científicos; aunque difieren en que los primeros siguen esquemas académicos o científicos y los indígenas fundamentan su sistema de constructos en la experiencia directa con los animales.

También se observaron diferencias en la relación de temor que los animales infunden a niños y niñas, siendo las niñas quienes presentan un mayor temor, además se vio un desconocimiento generalizado de la ubicación geográfica de varias de las especies que se presentaron a los participantes del primer estudio que no está presente en la población indígena. Los hallazgos se discuten desde la Biofília y se dan recomendaciones acerca de la importancia de diseñar programas de educación ambiental, que incorporen el conocimiento y protección de la fauna, a partir de los ecosistemas en los que se encuentren ubicadas las escuelas del país, y algunas de las experiencias de las comunidades indígenas.

\section{Palabras clave}

Teoría de los Constructos Personales, Relaciones humano-animal, Hipótesis de Biofília, actitudes hacia la fauna, saber indígena, Uitoto.

\section{Abstract}

This study was oriented to identify the bonds that children from different cultural contexts and regions from the majority Colombian society and an indigenous community from the Colombian Amazon develop toward animals through their personal constructs. Children form the majority society differ from the indigenous children in that the first group follow a conceptual structure based on ecological-scientific criteria while the indigenous sample follow their experiences with animals. There are also differences in the number of animals that girls are afraid of in comparison to boys in the first group. Children from Uitoto community were able to differentiate foreign animals easily than the first group of participants. Findings are discussed from the Biophilia hypothesis Recommendations are given about the importance of designing environmental education programs that incorporate the knowledge and protection of fauna from the ecosystems in which schools are located and some of the experiences of indigenous communities.

Key words

Personal Construct Theory, Human being-animal relationships, Biophilia Hypothesis, Attitudes toward animals, Indigenous knowledge, Uitoto

Artículo recibido el 31 de Mayo de 2010 y aprobado el 30 de Agosto de 2010.

1 Profesores de la Universidad Pedagógica Nacional. pdeparamo@gmail.com 


\section{Introducción}

Los vínculos afectivos con la naturaleza, y en particular con los animales, han sido cruciales en el proceso evolutivo de nuestra especie y en el bienestar físico y psicológico de las personas. Aunque se pueden observar distintos tipos de respuesta de carácter afectivo con el paisaje y las plantas, el lazo afectivo con los animales representa el principal vínculo con la naturaleza.

Los animales constituyen uno de los componentes naturales de mayor significado socioeconómico, científico y cultural de un país. El valor ambiental de los animales es tan importante como el de otros recursos naturales, igual que el agua, el suelo y los bosques. Tradicionalmente el valor asignado a los animales se relaciona con su importancia como medio de subsistencia, del cual la especie humana se ha valido para satisfacer sus necesidades de alimento y abrigo.

Hoy, este valor tiene un sentido diferente, se ve a los animales con otros intereses y apreciaciones desde el discurso de las diferentes disciplinas; entre ellas, el de la sociología, que ve la interacción entre humanos y especies no humanas como un reflejo de la condición humana, y justifica el examen cuidadoso de las actitudes de las personas hacia los animales, que permitiría llegar a importantes conclusiones sobre las normas sociales, la conducta y la estructura social (Kendall y Lobao 2004). Se suman a este tipo de estudios los que exploran la intersección entre el género con las actitudes hacia los animales y la manera como se han construido socialmente los conceptos de animal y naturaleza (Peek, Dunham, y Dietz, 1997).

Desde el punto de vista biológico se ha creado la necesidad de conocer los animales para identificar los estados biológicos y ambientales de un ecosistema, un río, o la supervivencia de una comunidad, pero también se da la necesidad de estudiar las interacciones humanas y no humanas para probar, de cierta manera, nuestros vínculos genéticos con la naturaleza, como lo sostienen Kellert y Wilson (1993), y Kahn, (2001), defensores de la Hipótesis de la Biofília, y Crawford y Krebs, (1998) de la psicología evolucionista.
En esta misma dirección, la ecología conductual estudia las relaciones evolutivas entre el comportamiento de una especie y su ambiente, donde la estructura social de dicha especie es un componente de la ecología conductual que explora los efectos del ambiente social sobre la evolución del comportamiento, incluyendo el comportamiento social humano (Alcock, 2001).

La psicología, además de hacer estudios comparados mediante la psicología evolucionista, estudia el papel que ejercen los animales sobre el componente afectivo del comportamiento humano; los animales han propiciado la protección y la seguridad de quienes los poseen, desarrollando la autoconfianza y la autoestima. Canosa y Minguell (2002) afirman por ejemplo que la convivencia con los animales despierta los sentimientos y actitudes más sensibles de cualquier persona; tener animales cerca conlleva a que los individuos experimenten mayor sensibilidad, ternura y nobleza.

Los estudios muestran que la convivencia con animales como perros, gatos, o peces, se constituye en compañía para mitigar los efectos de la soledad y el estrés (Flynn, 2000, Brown 2002); la investigación que aquí se resume tiene como punto de partida estas perspectivas psicológicas a partir de la exploración del sistema de constructos de los niños de la sociedad mayoritaria colombiana y de comunidades, también colombianas, como la Uitoto, en las que la apreciación y valoración por los animales son favorecidas por la interacción y afiliación continua con la naturaleza.

El concepto de constructo surgió de la Teoría de la Construcción Personal (TCP) propuesta por Kelly (1955), quien afirma (citado por Fransella, 1995) que el mundo es percibido por una persona en términos de los significados que la persona le aplique; el individuo crea sus propias maneras de ver el mundo en que vive y no es el mundo el que las crea para él. Las personas desarrollan modelos internos de la realidad con el fin de entender el mundo que los rodea, de la misma manera como los científicos desarrollan teorías.

Los constructos se construyen a partir de la observación y la experiencia; se definen mediante 
palabras y son particulares a los individuos por tener cada uno experiencias particulares, por lo que se denominan constructos personales; no obstante, muchas de nuestras experiencias son semejantes o compartidas, lo que lleva a la semejanza de unos constructos con otros.

En la medida en que una persona emplea un constructo de experiencia similar al empleado por otra, sus procesos psicológicos son similares a los de esa otra persona. Cuando valoramos una persona como atractiva, arrogante e inteligente, estamos haciendo uso de constructos que hemos aprendido para describir o explicar su comportamiento. Los constructos se organizan en sistemas; grupos de constructos que encierran relaciones superordenadas y subordinadas.

Los constructos se usan además para la predicción de lo que viene, y la experiencia confirma o no tales predicciones haciendo que éstos evolucionen y puedan eventualmente modificar todo el sistema de constructos; al explorar el sistema de constructos utilizados por las personas, se puede entender su forma de actuar. Los estudios que aquí se describen fueron emprendidos a partir del interés por explorar el sistema de constructos de los niños de la sociedad mayoritaria e indígena colombiana sobre los animales, y ver si se diferencian entre sí.

Tal como lo han señalado autores como Strong (1995) y Shepard (1996), el mundo moderno ha venido perdiendo las conexiones satisfactorias con la naturaleza, razón por la cual resulta importante mirar la diversidad cultural como un medio de entender y restablecer dichas conexiones, reconociendo sus fuertes vínculos en todos los aspectos de sus vidas.

Lastimosamente el conocimiento sobre los animales de la región es escaso en el país. Se citan estudios científicos que presentan resultados técnicos, y que por lo mismo también presentan cierto grado de dificultad para ser entendidos por el público en general; a esto se suma la transformación conceptual en la que hemos estado inmersos a través de los años, generalmente en el papel de distinguir y reconocer animales, inspirada por medios comunicativos como la televisión y el cine, así como por sistemas educativos de otros países, mediante libros y textos escolares.

Este tipo de material ha facilitado de alguna manera el conocimiento de los animales, permitiendo que la relación que sostenemos con ellos sea armónica y afectiva. Sin embargo, hay que advertir que esa relación de sensibilidad está dada, en la mayoría de los casos, únicamente con especies foráneas, es decir con animales del norte de América y de África, y en pocas, por no decir ninguna, ocasiones con la fauna latinoamericana, particularmente colombiana, que cuenta con muy poca difusión.

Es cierto que en Colombia durante los últimos años se vienen haciendo algunos estudios relacionados con el uso y manejo de la fauna (Campos, Ulloa y Rubio, 1996; Mateos, 1998; Páramo, Arias, Melo, Pradilla y Pabón, 1999; Barraza y Ceja, 2001; Nassar, 2001; y Ulloa, 2002), pero es necesario iniciar trabajos orientados a conocer el pensamiento de los niños respecto de su relación y vinculación con la naturaleza y los animales: conocimiento, actitudes, formas de relación, sensibilidad ante los problemas que enfrentan, etc. Para la pedagogía y el desarrollo de los programas de educación ambiental es importante partir del conocimiento de los niños, pues son ellos quienes en últimas podrán canalizar hacia el futuro el desarrollo de propuestas de vida armónica con la naturaleza.

Bajo el contexto de conocimiento tradicional, y de la relación persona/naturaleza, es que esta investigación se propuso explorar y analizar la forma como los niños conceptualizan a los animales desde su contexto cultural. La comunidad indígena Uitoto, seleccionada para esta investigación, es una de las mejor distribuidas en la selva amazónica colombiana, y cuenta con un amplio conocimiento sobre los animales, que son reconocidos por los Uitoto como un elemento indispensable de subsistencia, más que eso, los consideran como esenciales para el desarrollo simbólico y cultural de sus niños.

A partir de esta justificación se formularon las siguientes interrogantes: ¿A qué constructos recurren los niños para caracterizar los animales?; ¿diferencian los niños de las distintas comunidades los animales locales de los foráneos?; ¿ existen diferen- 
cias de género en la conceptualización que se hace sobre los animales? Y por último, ¿qué discrepancia de constructos sobre los animales existen entre los niños indígenas Uitoto y los niños no indígenas de la sociedad mayoritaria colombiana?

\section{Metodología}

La aproximación al objeto de estudio fue de carácter descriptivo exploratorio, y se concentró principalmente en recoger las conceptualizaciones de los niños de las sociedades mayoritarias y de la comunidad indígena Uitoto colombiana, mediante un tipo de entrevista llevada a cabo a partir del sorteo libre de tarjetas que contenían las imágenes de distintas especies animales.

\section{Participantes}

La investigación se llevó a cabo mediante dos estudios independientes pero con el mismo propósito y procedimiento; en el primer estudio ${ }^{2}$ se entrevistaron niños de la sociedad mayoritaria ${ }^{3}$ de las áreas rurales de los municipios de Leticia (Amazonas), Lloró (Chocó), San José del Guaviare (Guaviare) y Minca (Sierra Nevada de Santa Marta), un total de 240 personas. Por otro lado, y guardando las mismas proporciones, se entrevistaron 60 niños del área urbana de Bogotá y de sus municipios aledaños (La Vega, Subachoque y Guasca en Cundinamarca), conformando una muestra total de 300 participantes de ambos sexos y con edades que fluctuaban entre los 9 años y 17 años. Las regiones fueron seleccionadas con el criterio de tomar una muestra de diferentes pisos térmicos y por consiguiente biomas en donde las personas tienen contacto con diversas especies animales.

2 La información sobre la población de la sociedad mayoritaria se basó en el estudio con niños campesinos y urbanos realizado por Páramo, P.; Arias, J. D.; Melo, M. C.; Pradilla, H. y Pabón, C. (1999). Nuestros vínculos con los animales. Universidad Pedagógica Nacional.

3 Por sociedad mayoritaria se entiende aquel grupo social que siendo mayoría en número dentro de la sociedad colombiana comparte igualmente prácticas sociales, lengua, tradiciones, y demás características políticas y culturales que le diferencian de otros grupos 0 naciones minoritarias con una autoidentificación étnica, $y$ que se han formado a lo largo de un proceso histórico cuyo resultado hace que sus miembros presenten comunidad de lengua, organización sociopolítica, de economía y de cultura sobre la base de un asentamiento en un territorio propio común, y que son igualmente reconocidos jurídicamente por el Estado Colombiano. Luis Guillermo Vasco, 1982, “Nacionalidades Minoritarias en Colombia". Mimeo. Bogotá.
El segundo estudio ${ }^{4}$ se llevó a cabo con niños de la comunidad indígena Uitoto, pertenecientes a los grupos familiares de Puerto Belén, Puerto Pizarro y Los Estrechos, la etnia está constituida por aproximadamente 6.000 personas que habitan la zona selvática del medio río Caquetá, Departamentos del Amazonas y Caquetá. El grupo seleccionado para la investigación estuvo constituido por 35 niños de ambos sexos, con edades que oscilaron los 7 y 13 años.

\section{Instrumento}

Se utilizaron 21 fotografías de animales vertebrados de diferente clase (aves, peces, mamíferos, reptiles), que en su mayoría pertenecían a especies reconocidas de ecosistemas colombianos y foráneos de origen africano o asiático. Otro aspecto que se consideró para seleccionar las fotografías, fue incluir especímenes con algún riesgo de extinción, como el Oso de Anteojos, el Tigre, el Cóndor, el Delfín Rosado, entre otros, para facilitar el reconocimiento y la posterior sistematización de la información; las fotografías se numeraron al respaldo.

A diferencia de los niños de la sociedad mayoritaria, la investigación con los niños Uitoto se desarrolló utilizando tarjetas con dibujos de animales vertebrados propios de la región amazónica en la que habitan; las tarjetas se diseñaron con esquemas tomados de investigaciones relacionadas con inventarios de fauna colombiana y latinoamericana, como las de Eisenberg (1989), Emmons y Feer (1990), López y Suerote (1993), De Civrieux (1974) y Townsend (1984).

La razón por la que se escogieron animales vertebrados obedeció a que en la mayoría de los trabajos sobre percepción de los animales se ha encontrado que las personas prefieren exhibir sus preferencias estéticas, emocionalidad, conciencia y atracción hacia las especies vertebradas grandes.

\section{Procedimiento}

La información sobre la conceptualización que tienen los niños de la sociedad mayoritaria e indígena acerca de los animales fue recogida mediante el modelo de entrevista individual, denominado Clasificación

4 Datos tomados de la investigación realizada por Galvis y Tovar (2007) con niños indígenas Uitoto. 
Múltiple de Îtems o CMI (Canter, Brown y Groat, 1985; Breakwell y Canter, 1993; Feixas, 1995, 2003; Páramo 2008). La principal ventaja de este modelo está en que permite el surgimiento libre y espontáneo de criterios de los participantes para realizar clasificaciones sobre los elementos a clasificar, en este caso las fotografías o dibujos de los animales, buscando con ello que el investigador interfiera lo menos posible con sus preconceptos en dichas clasificaciones, como suele ocurrir cuando se hace uso de cuestionarios.

\section{Instrucciones}

Para orientar la entrevista mediante la CMI los participantes fueron entrevistados individualmente, se les solicitó que hicieran inicialmente clasificaciones libres y luego algunas dirigidas, siguiendo las siguientes instrucciones:

Estamos haciendo un estudio acerca de lo que las personas piensan y sienten sobre estas fotografías/ dibujos; en ellas se presenta una serie de animales, por favor obsérvelas. Ahora, clasifique las fotografías/dibujos en grupos siguiendo algún criterio, de manera que las fotos/dibujos ubicadas dentro de cada grupo tengan algo similar o en común, además, cada grupo de fotografías/dibujos debe diferenciarse de los otros grupos y llevar un nombre distinto. Usted puede elegir cualquier criterio que quiera para agrupar estas fotografías y puede dividirlas en tantos grupos cuantos quiera. No hay tipo alguno de clasificación correcta o incorrecta para sus respuestas, lo que cuenta es su opinión. Tómese el tiempo necesario y siéntase en libertad de hacer los comentarios que quiera.
Una vez terminada la primera clasificación se le preguntaba al niño participante por el criterio utilizado para agrupar los elementos y dar los nombres a cada grupo a través de las siguientes instrucciones:

\begin{abstract}
Ahora, dígame las razones de su clasificación, ¿qué tipo de criterio utilizó y qué es lo que tienen en común las fotografías y dibujos en cada grupo? $¿$ Cree que puede hacerlo de nuevo en otra forma? Si cree que es posible, comience a clasificarlas de nuevo y siéntase en libertad de decirme lo que quiera a medida que las va clasificando.
\end{abstract}

Después de tres clasificaciones cumplidas, o si el niño expresaba no poder hacer más clasificaciones, se daban las siguientes instrucciones como introducción a una serie de preguntas que inducían a realizar clasificaciones dirigidas:

Usted ya está familiarizado con las fotografías y/o dibujos, ahora agrúpelas de acuerdo a:

a. Qué tan agradables le parecen cada uno de los animales.

b. Cuán importantes son.

c. Cuál es su ubicación geográfica.

d. Si considera que el animal se encuentra en peligro de desaparecer.

La información resultante de estas clasificaciones libres fue recogida en el formato que se presenta en la Tabla 1, ilustrando con un ejemplo la manera como fue consignada la información de cada niño participante:

Tabla 1. Registro de la información (ejemplo)

\begin{tabular}{|l|l|c|c|}
\hline Criterio/Categorias & Clasificación & $\begin{array}{c}\text { Fotografias y dibujos que correspondieron } \\
\text { a las clasificaciones hechas por los niños. }\end{array}$ & $\begin{array}{c}\text { Observaciones del } \\
\text { participante }\end{array}$ \\
\hline Hábitat & Viven en el aire & $1,2,5,7,9,10$ & \\
\hline & Viven en el agua & $3,11,15,16,19,20$ & \\
\hline & Viven en la tierra & $4,6,8,12,13,14,17,18,21$ & \\
\hline Clase & Mamiferos & $4,6,8,12,13,12,17$ & \\
\hline & Aves & $1,2,5,7,9,10$ & \\
\hline & Peces & $4,11,15,16,19$ & \\
\hline & Reptiles & 18,21 & \\
\hline
\end{tabular}


La sistematización de la información acumulada mediante este tipo de entrevistas se llevó a cabo mediante el análisis de contenido de los constructos empleados por los participantes y mediante el apoyo en un software denominado Análisis de Escalogramas Multidimensionales, mejor conocido por sus siglas MSA (Borg y Groenen, 2005). Este programa, alimentado por una matriz sobre las distintas clasificaciones que hicieron los niños, arroja como resultado unos gráficos que muestran en un espacio bidimensional una serie de puntos que representan los animales que fueron objeto de la entrevista.

La distribución de estos puntos representa el grado de correlación entre los perfiles producto de las agrupaciones creadas por los participantes con los animales. Los investigadores procedieron a crear unas particiones o regiones sobre las distancias espaciales, entre cada perfil a que da lugar cada animal, producto de las agupaciones en las que es incluido cada vez que se hace una clasificación, para explicar las asociaciones entre los distintos animales e identificar así el sistema conceptual de los niños sobre los animales en términos de las similitudes o diferencias entre ellos.

\section{Resultados}

En aras de la brevedad que exige la revista para presentar tal cantidad de información, se omiten las matrices de datos que permitieron sistematizar las clasificaciones de los participantes y los gráficos que arrojan los programas MSA al procesar dichas matrices 5 . Más adelante se presentan, a manera de ejemplo ilustrativo, algunos de los gráficos que arrojó el programa MSA, producto de las entrevistas a los niños de las comunidades campesinas y Uitoto.

Nos limitaremos a comentar y analizar los principales hallazgos de los dos estudios, fruto de las clasificaciones libres y de las preguntas dirigidas, a partir de la perspectiva evolucionista de la psicología a la que ya hicimos referencia, y en particular desde la mirada del trabajo de Kellert sobre Biofília.

5 Los entusiastas pueden encontrar información detallada de cada trabajo de investigación en los informes de estas investigaciones citados en las notas 3 y 4
Para analizar los constructos de los que se valieron de forma libre los participantes, de lo que hemos denominado sociedad mayoritaria (campesinos y citadinos) y minorías étnicas (niños de la comunidad Uitoto) para clasificar las distintas imágenes de animales, se tuvo en cuenta la tipología que propone Kellert (1997), en un intento por mostrar los fundamentos biológicos de los valores humanos asociados a los elementos de la naturaleza, y que según la Biofília (Kellert y Wilson, 1993) soportarían la hipótesis que establece la necesidad humana para afiliarse con el resto de los elementos vivos y los procesos asociados con la vida. Esta tipología es por supuesto otro sistema de constructos elaborados desde una teoría científica, en este caso la Biofília, y se recurre a ella para interpretar el sistema de constructos de los participantes del estudio.

Los valores de Kellert sobre la relación con la naturaleza son: el utilitarista (su relación con la naturaleza es de uso y explotación; el naturalista (quien experimenta satisfacción de la experiencia directa o del contacto con la naturaleza); el ecológico-científico, el estético (que ve la belleza de la naturaleza); el simbólico (que utiliza la naturaleza para expresiones metafóricas a través del lenguaje); el humanístico (con apego emocional o amor por la naturaleza); el moralista (con una preocupación ética por la naturaleza); el de dominancia (ejercer control físico por la naturaleza) y el negativismo (que involucra los sentimientos de temor que hacia algunos elementos de la naturaleza). Los proponentes de la Biofília, sostienen la posibilidad de que estas valoraciones o afiliaciones con la naturaleza están arraigadas en nuestra biología y por consiguiente hacen parte de la naturaleza humana.

\section{Los niños de la sociedad mayoritaria y su conceptualización acerca de los animales}

\section{Clasificaciones libres}

A continuación se presenta una descripción y el análisis de los datos generales del grupo de niños de la sociedad mayoritaria. En primer lugar aparece la tabla que recoge las distintas categorías de clasificación de las que se valieron los participantes, después 
de someterlas a un Análisis de Contenido (Ocampo, 2008); para llevar a cabo este análisis se crearon categorías que pudieran agrupar los constructos empleados por los participantes al ir realizando las agrupaciones.

El análisis de contenido realizado entre las distintas regiones no muestra diferencias sobresa- lientes en los conceptos empleados por los distintos grupos de la sociedad mayoritaria de las diferentes regiones del país (ver Nota 4). La Tabla 2 describe cada una de las categorías en porcentajes relativos, éstas fueron creadas por los investigadores con base en las agrupaciones de las clasificaciones libres de los niños.

Tabla 2. Análisis de contenido de las clasificaciones hechas por los niños de la sociedad mayoritaria

\begin{tabular}{|c|c|c|}
\hline $\begin{array}{l}\text { Categorías análisis } \\
\text { de contenido }\end{array}$ & Clasificaciones de los niños & $\%$ \\
\hline Hábitat & $\begin{array}{l}\text { Lugar en el que habitan los animales: viven en la tierra, viven en el aire, viven en los palos } \\
\text { o viven en el agua, viven en la casa. }\end{array}$ & 22,1 \\
\hline Clase & Reconocimiento taxonómico: si son mamíferos, aves, peces o reptiles. & 20,5 \\
\hline Alimentación & Tipo de alimentación: carnívoros, herbívoros y omnívoros, comen insectos, comen frutas. & 7,2 \\
\hline Utilidad & $\begin{array}{l}\text { Tipo de uso que prestan al hombre: ningún beneficio, para consumo, de seguridad, com- } \\
\text { pañía, se comercializan, ayudan al trabajo. }\end{array}$ & 6,9 \\
\hline Doméstico/salvaje & Animales con domesticación hecha por el hombre. & 6,2 \\
\hline Características físicas & Morfología externa de los animales: color de las plumas o de la piel de los animales. & 6,1 \\
\hline Mecanismos de supervivencia & Mecanismos de adaptación natural de los animales: indefensos, se camuflan. & 5,8 \\
\hline Número de extremidades & Número de extremidades: bípedos, cuadrúpedos o no tienen extremidades. & 4,8 \\
\hline Piel & Forma de la piel: tienen pelo, tienen plumas, tienen caparazón, tienen cuero, tienen escamas. & 3,8 \\
\hline Reproducción & Sistema reproductivo de los animales: reproducción por huevos, mamíferos. & 3,2 \\
\hline Locomoción & Forma como se mueven los animales: vuelan, saltan, nadan, se arrastran. & 3,2 \\
\hline Peso y tamaño & $\begin{array}{l}\text { Descripción de los animales por su tamaño y volumen: pequeños, o grandes; o por su } \\
\text { peso: pesados, livianos. }\end{array}$ & 2,3 \\
\hline Ubicación geográfica & Distinción regional: son de la región, no son de la región. & 1,4 \\
\hline Peligro de extinción & $\begin{array}{l}\text { Criterio de reconocimiento de riesgo de extinción de un animal: están o no en peligro de } \\
\text { extinción. }\end{array}$ & 1,13 \\
\hline Habilidad & Velocidad de desplazamiento: lentos, ágiles, rápidos o trepan árboles. & 1 \\
\hline Agrado & $\begin{array}{l}\text { Emocionalidad asociada con los animales: si generan o no repulsión, me gustan, no me } \\
\text { gustan. }\end{array}$ & 0,7 \\
\hline Cadena alimenticia & $\begin{array}{l}\text { Intercambio de energía entre especies: según lo que comen o según al animal que se } \\
\text { coman, depredador, no depredador. }\end{array}$ & 0,6 \\
\hline Comunes/poco comunes & Grado de familiaridad: conocidos, no conocidos. & 0,6 \\
\hline Peligrosidad & $\begin{array}{l}\text { Grado de riesgo generado el ser humano: pican, tienen veneno, muerden, o se lo comen } \\
\text { a uno, inspiran temor, son bravos, son mansos, son inofensivos. }\end{array}$ & 0,53 \\
\hline Orden & $\begin{array}{l}\text { Clasificación de un animal de acuerdo a las características de similitud entre especies: } \\
\text { primates, roedores. }\end{array}$ & 0,33 \\
\hline Especie & Nombre común del animal: perro, elefante, conejo, etc. & 0,3 \\
\hline Inteligencia & $\begin{array}{l}\text { Criterios de similitud al humano: parecidos al hombre, no parecidos, inteligentes, no muy } \\
\text { inteligentes. }\end{array}$ & 0,2 \\
\hline Conocidos/poco conocidos & Grado de conocimiento de un animal. & 0,2 \\
\hline Característica circense & $\begin{array}{l}\text { Reconocimiento de un animal que trabaja en espectáculos circenses: los han visto o no } \\
\text { en los circos. }\end{array}$ & 0,08 \\
\hline Sangre fría/sangre caliente & Clasificación de un animal por su tipo de sangre. & 0,02 \\
\hline
\end{tabular}


De los nueve valores propuestos por Kellert, la mayor parte de las categorías empleadas por los niños participantes de este estudio, como el hábitat, la clase, el tipo de alimentación, el filum, los mecanismos de supervivencia empleados por las especies, el número de extremidades, el sistema reproductivo, características físicas, etc., corresponderían a valoraciones humanas ecológico-científicas que cumplen una función adaptativa ligada al conocimiento y entendimiento sobre la naturaleza.

Otras categorías que corresponderían al valor humanista son: las que diferencian los animales entre domésticos o salvajes, el peligro de extinción que enfrentan algunas de las especies, la capacidad intelectual de algunos, la defensa de sus crías, la socialización o las características circenses; que responderían a la necesidad de estar vinculado a un grupo social, el compartir o a la necesidad de tener compañía; otras, como la utilidad que representan las especies para el hombre, corresponderían al valor utilitario que menciona el autor, que respondería igualmente a la necesidad del sustento físico a partir de percibir la naturaleza como un recurso necesario para la subsistencia.

Los criterios de "comunes o poco comunes" y "conocidos o desconocidos", podrían formar parte del valor naturalista, en la medida en que responden a la curiosidad y contribuirían al desarrollo físico y mental del individuo; finalmente, el negativismo podría reflejarse en clasificaciones como "agrado/ desagrado" o "peligrosos/no peligrosos", que responderían a la necesidad de seguridad o protección.

Así como la Hipótesis de la Biofília establece el valor adaptativo de acercarnos y contemplar la naturaleza, de igual manera postula que existen biofobias que pudieron cumplir un valor adaptativo en nuestra especie y que aún se mantienen como vestigios de nuestro pasado evolutivo. La biofobia se refiere a las respuestas fóbicas a ciertos estímulos naturales que presumiblemente han constituido una amenaza a la evolución humana (Ulrich, 1993). No figuran categorías relacionadas con los valores de: estética, simbolismo, moralista o dominio.

Aunque en todos los niños predomina una estructura de conocimiento de los animales a partir de valores ecológico-científicos, no significa que posean una estructura ecológica clara de interdependencia entre las especies y el hábitat. Durante las entrevistas no fueron muy frecuentes los comentarios relativos a cadenas alimenticias, intercambio de energía, o funciones de las especies dentro de nichos.

Prueba de ello es que la valoración acerca de la importancia de las especies se juzga en términos de la utilidad que prestan, antes que en términos del sostenimiento de los ecosistemas. Además, resulta preocupante que dentro de las clasificaciones libres aparezcan índices muy bajos, en todos los grupos de niños de la sociedad mayoritaria en las distintas zonas del país, de criterios que denoten valores relacionados con la conservación de las especies, a pesar de que se presentan fotografías de algunas que están en vía de extinción, e incluso, la fotografía de una Ballena que está siendo desollada.

Figura 1. M.S.A., de uno de los grupos de la sociedad mayoritaria colombiana

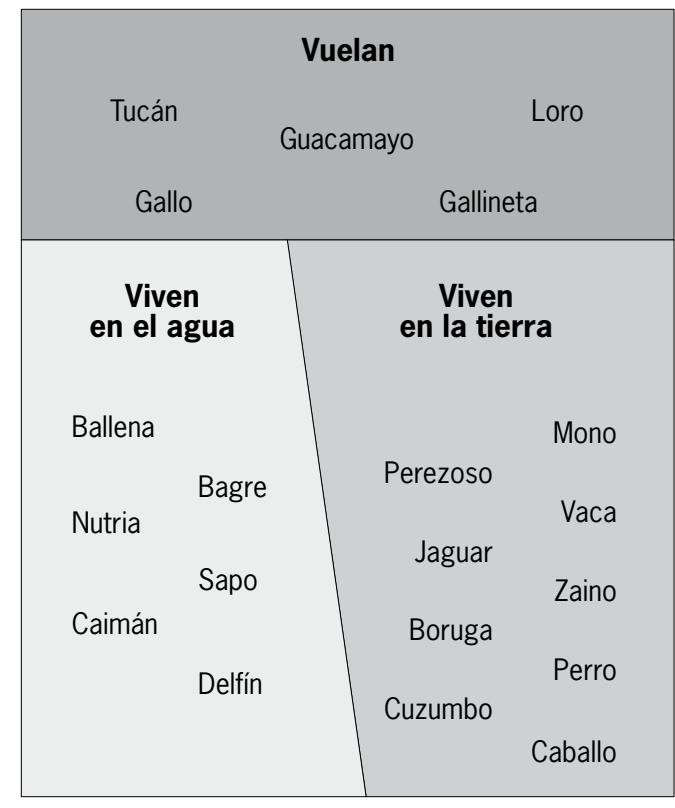

Los constructos más frecuentemente empleados por los niños de la sociedad mayoritaria para clasificar los animales, reflejan igualmente la influencia educativa: los participantes entrevistados de este primer estudio son niños formados académicamente en sistemas educativos muy similares, a pesar de las diferencias regionales. Independientemente de las experiencias directas y diferentes que puedan haber 
tenido los niños de estas regiones, prevalecen las categorizaciones científicas de un sistema educativo general.

Quizás por esto no aparecen las categorías valorativas, moralistas o simbólicas, a las que se refiere Kellert (1997), lo que justificó ampliar la perspectiva del estudio con la inclusión de niños que hacen parte de sistemas educativos diferentes, como las comunidades indígenas, quienes además de ser formados con currículos diferentes y atribuir mayor simbolismo a la fauna, tienen un contacto más directo con la naturaleza y en particular con los animales.

\section{Clasificaciones dirigidas}

Con respecto a las preguntas dirigidas (agrado, importancia, extinción y ubicación geográfica) se pudo observar en primer lugar que, el agrado por los animales está ligado aparentemente a la docilidad, familiaridad o cercanía de las especies con el hombre, apariencia, y a la publicidad que se hace con respecto a algunas especies; mientras que se agrupó como desagradables principalmente a los animales asociados con el peligro, y aquellos con los que no se tiene un contacto frecuente $o$ son menos publicitados. Por esto predominan entre los más agradables la Vaca, el Perro, la Guacamaya, la Tortuga y el Delfín y como más desagradables la Piraña, el Tiburón, el Gorila y la Serpiente.

La importancia de las especies está determinada principalmente por su carácter utilitarista: los evaluados como más importantes son los que sirven para el trabajo o la alimentación, y los menos importantes son los salvajes o peligrosos, que según los participantes prestan "poca utilidad", como por ejemplo: la Culebra, el Tigrillo, la Raya, la Boa, el Gorila, la Fara, etc.

En cuanto a la ubicación geográfica, la mayor parte de los participantes en el estudio no reconoció los animales marinos o acuáticos como parte de la región (ubicados en la clasificación como locales o de la región), tal es el caso de la Ballena o el Tiburón, y por lo general tampoco reconocen muy bien las especies que se encuentran en distintas regiones del país, como el Oso de Anteojos. Aunque la mayor parte de los niños entrevistados identifican buena parte de las especies de otros continentes como foráneas, y sin embargo también creen que algunas especies como el Gorila, el León o el Tigre son propias de la región.

Esto también se puede atribuir a que la enseñanza de los recursos se centra en contenidos de libros que no informan acerca de nuestros recursos, descuidando las experiencias del entorno inmediato como medio de aprendizaje. No obstante ante la pregunta se observaron diferencias regionales importantes, en particular respecto de los animales reconocidos como de la localidad, los grupos de los Llanos, la Sierra Nevada de Santa marta y el Amazonas, hicieron clasificaciones bastante acertadas de las especies de la localidad.

Finalmente, la pregunta sobre los animales en peligro de extinción, la investigación hizo evidente una gran carencia de información al respecto en todos los niveles educativos, los estudiantes no reciben información relacionada con esta problemática ambiental; aunque se presentan algunas excepciones, como el reconocimiento de los grupos de cuarto y séptimo grado de Minca (Sierra Nevada de Santa Marta) sobre el peligro que enfrenta la Guartinaja, que refleja la influencia del programa de recuperación de esta especie que se ha desarrollado en la región.

Figura 2. M.S.A., de la pregunta por el agrado a los niños de la sociedad mayoritaria colombiana

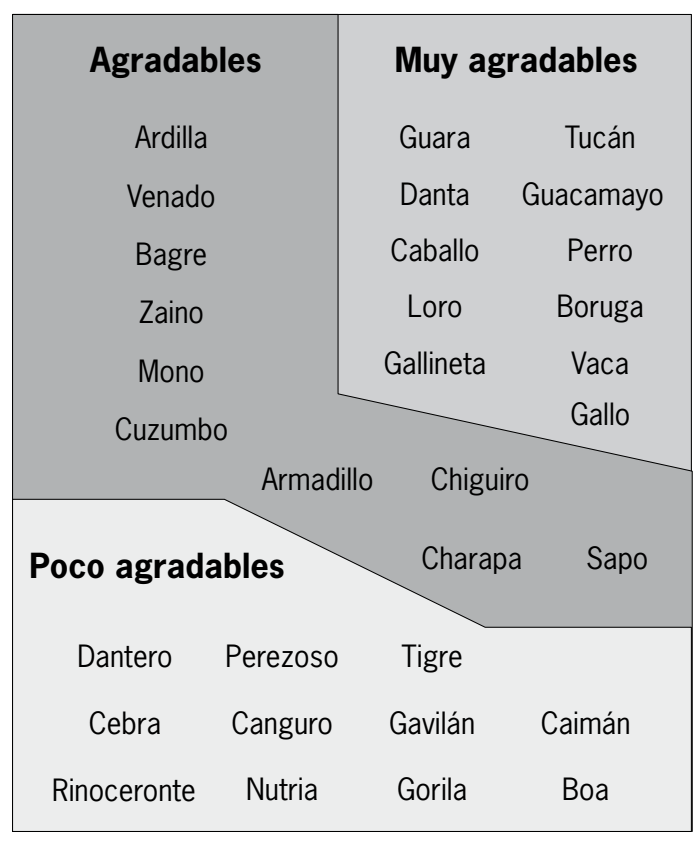


Con el propósito de estudiar las diferencias de género, en la percepción sobre los animales en las cinco regiones estudiadas, se consideró la información recopilada a partir de las clasificaciones hechas sobre la pregunta dirigida de "Agrado"; los participantes hombres difieren de las mujeres a partir de la cantidad de animales que consideran desagradables. Una explicación para estos resultados se puede atribuir a que, por lo general, las niñas exploran menos el ambiente, tienen menos contacto con los animales y aprenden de forma vicaria el rechazo a un mayor número de ellos; los niños, por el contrario, son motivados a tener mayor diversidad de mascotas y no tener miedo a los animales; aquellos que muestran temor por algún animal son tratados como cobardes y comparados con las niñas.

En términos generales se puede afirmar que la conceptualización sobre los animales por parte de la población joven, campesina y de la ciudad, está estructurada principalmente a partir valoraciones ecológico-científicas, como el hábitat de los animales y el tipo o clase de especie. No se observaron diferencias importantes entre regiones pero sí de género.

\section{Los niños de la comunidad indígena Uitoto y su conceptualización acerca de los animales}

\section{Clasificaciones libres}

El conjunto de ítems para ser clasificados por este grupo incluyó la Boruga, el Sapo Berrugoso, el Cuzumbe, el Tigre Dantero, el Chiguiro, el Zaino, el Perezoso, el Venado, la Nutria, el Guara, la Ardilla, el Mico Maicero, la Danta, el Caimán, la Charapa, el Armadillo, el Gavilán Veloz, la Gallineta, el Loro Churuquero, el Guacamayo, el Tucán, el Caballo, la Vaca, el Perro, la Gallina, la Boa, el Bagre, el Canguro, el Rinoceronte, el Gorila y la Cebra.

Se procedió de la misma manera que con el grupo de la sociedad mayoritaria para sistematizar las clasificaciones hechas por los niños de la comunidad Uitoto. Las diferentes clasificaciones se recogen en la Tabla 3, que agrupa los criterios o constructos empleados por los participantes y sus porcentajes relativos para agrupar los dibujos de los animales, después del Análisis de Contenido que hicieron los investigadores a partir de las distintas agrupaciones de los niños.

Tabla 3. Análisis de contenido de las clasificaciones hechas por los niños indígenas Uitoto

\begin{tabular}{|l|l|c|}
\hline \multicolumn{1}{|c|}{$\begin{array}{c}\text { Categorías análisis } \\
\text { de contenido }\end{array}$} & \multicolumn{1}{|c|}{ Clasificaciones de los niños } & $\%$ \\
\hline Clase & Clasificación taxonómica de los animales: pájaros, mamíferos, carrasposos. & 25 \\
\hline Tipo de alimento que consumen & $\begin{array}{l}\text { Se refiere a la fuente principal de alimentación del animal: los que comen carne, pepas, frutas, } \\
\text { insectos, gusanos, hierbas, los que comen peces. }\end{array}$ & 20 \\
\hline Hábitat & Se refiere a los espacios en los que viven los animales: en la casa, en el monte, en el río. & 20 \\
\hline Locomoción & Forma de desplazarse el animal: los que vuelan, caminan, reptan, nadan, trepan y se arrastran. & 15 \\
\hline Morfología & $\begin{array}{l}\text { Descripción física del animal: formas de las patas, de las colas, con pico y alas, con dientes, } \\
\text { con aletas. }\end{array}$ & 13 \\
\hline Sonidos emitidos & Voces con los que se comunican: aúllan, pían, rugen. & 7 \\
\hline
\end{tabular}

Las clasificaciones de los niños muestran una estructura conceptual para distinguir y reconocer a los animales a partir de la clase, con un $25 \%$, el hábitat, con un $20 \%$, la fuente de alimentación, con $20 \%$, la locomoción con $15 \%$, su morfología con $13 \%$ y mediante los sonidos que emiten los animales, con el 7\%. Al igual que lo observado con los integrantes del primer grupo, las categorías se ubican dentro de las valoraciones ecológico-científicas de Kellert, que cumplen un valor adaptativo para el reconocimiento de las especies.

Para el caso de los niños y su conceptualización de los animales, estos criterios están dados por el conocimiento ecológico, pues la mayoría de las 
clasificaciones estuvieron dirigidas a describir el hábitat, el alimento, las características físicas y las clases de animales. El criterio utilitarista es evidente en los niños, ya que la mayoría de animales descritos y categorizados tienen un valor de uso y servicio para la comunidad.

Lo que llama la atención al comparar los dos sistemas conceptuales es la aparición de la categoría "sonidos" emitidos por los animales, que cumple un papel muy importante en la identificación del animal en el ambiente selvático; otro aspecto notorio es que no aparece la distinción entre animales domésticos y salvajes, ni la de animales peligrosos y no peligrosos, como si apareció en los grupos de la ciudad y campesinos de distintas regiones del país. Resulta difícil atribuir estos resultados al currículo, pues aunque sí existe una escuela, no hay un currículo estructurado para estas comunidades, y la información sobre la fauna no hace parte de los contenidos que abordan las maestras.

Contrario a lo esperado, no figuran categorizaciones de los animales a partir de su simbolismo o significado para facilitar la comunicación o el pensamiento de la comunidad, como tampoco clasificaciones que impliquen valores morales, de significado espiritual, orden o armonía con la naturaleza.

Figura 3. M.S.A., del grupo de niños Uitoto

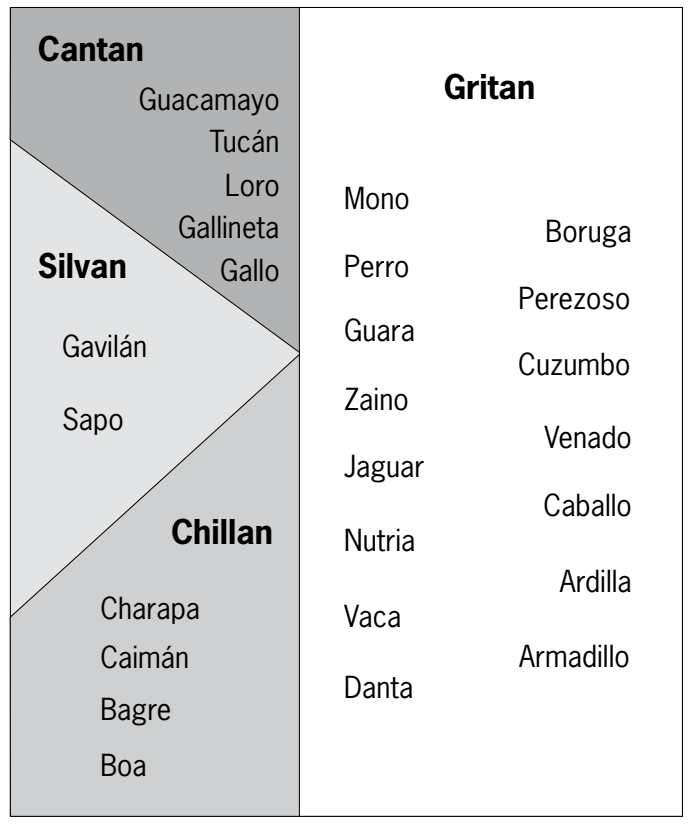

Figura 4. M.S.A., del grupo de niños Uitoto

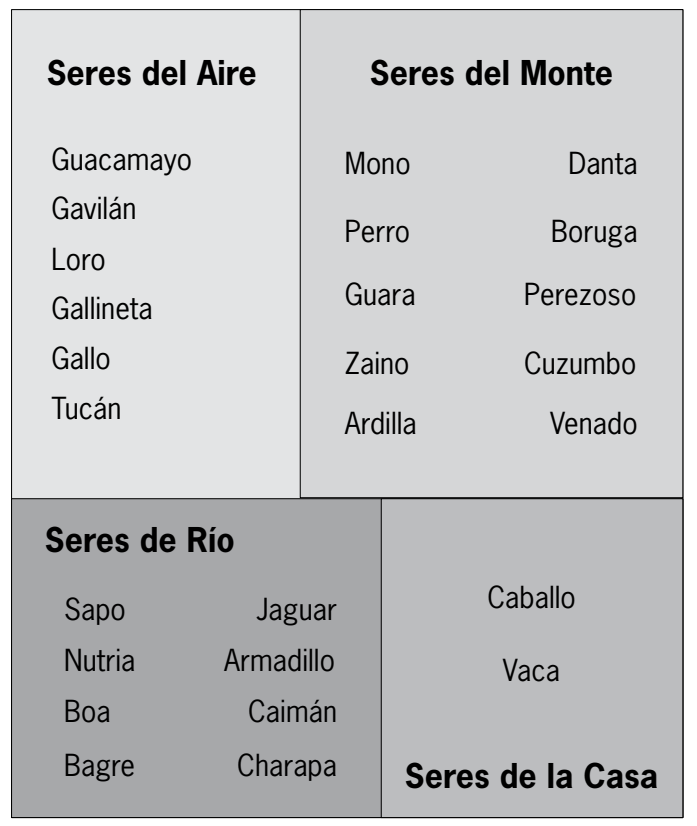

\section{Clasificaciones dirigidas}

Después de las clasificaciones libres, se dirigieron preguntas a cada uno de los participantes para establecer el grado de apreciación hacia los animales en torno a la relación con el agrado, así como también el reconocimiento y la presencia de las distintas especies en el territorio. Respecto a la pregunta sobre el agrado, fueron considerados agradables aquellos que prestan un servicio utilitario a la comunidad, porque son importantes cuando proporcionan materiales para la elaboración de instrumentos y adornos como plumas y picos.

Los cantos y sonidos de algunos animales también son tenidos en cuenta, gracias a que se involucran en la formación de los niños como cazadores; animales como la Boruga, el Venado, la Guara, el Chigüiro, la Danta, el Saino y la Gallineta son fuente importante de alimento; mientras que animales como la Vaca, el Caballo y el Perro son apreciados por su utilidad en las labores sociales de la aldea; el Armadillo es muy agradable para los niños porque sirve como instrumento de entrenamiento para que los cazadores adultos inicien a los niños varones en las faenas de cacería.

Aparentemente las niñas comparten los mismos gustos de los niños, pero difieren en que presentan 
más agrado por los animales que aportan alimentos como la Danta, la Guara, la Boruga, el Venado, el Chigüiro, la Gallineta y el Saino. La Boa fue la única especie clasificada como desagradable, debido al peligro que representan estos animales para las personas.

En cuanto a la localización geográfica, los participantes coinciden en la identificación de las especies que habitan en su territorio y cuales no; para ellos existe una diferencia marcada entre los animales foráneos y locales. La mayoría de niños estuvo de acuerdo en afirmar que las especies animales presentadas en las tarjetas con dibujos de animales como la Cebra, el Gorila, el Canguro y la Cebra no son animales de la región, que les resultaban desconocidos, y no pertenecen al monte.

Finalmente, no se observaron diferencias de género en cuanto a preferencias y conocimientos sobre la fauna; se mantiene una homogeneidad constante entre los niños y niñas en su gusto, agrado y rechazo por los animales, tanto por la especie como con la cantidad de animales considerados agradables y desagradables.

En síntesis, los niños Uitoto estructuran su conocimiento sobre la fauna a partir de criterios ecológicos dentro de los que predomina la clase, el tipo de alimento, el hábitat, la locomoción, la piel y los sonidos que emiten; vale la pena destacar que si bien estos conceptos hacen parte de la misma valoración ecológica-científica propuesta por Kellert, los criterios parecen estar mucho más relacionados con la experiencia directa que tienen con la fauna local. Tal es el caso por ejemplo de la clasificación sobre el tipo de piel de los anfibios al que denominaron carrasposo; también el tipo de alimento que consumen, el hábitat, que es descrito más específicamente de lo que hicieron los niños del primer grupo: animales de la casa, del monte, del río, y el tipo de sonidos que emiten (aúlla, rugen).

\section{Discusión}

La información arrojada por estos dos estudios es importante, por cuanto no se conocen muchos trabajos que den cuenta del conocimiento de los niños colombianos sobre los animales, y mucho menos acerca las conceptualizaciones o constructos de los que se valen los niños de comunidades indígenas. La mayoría de investigaciones sobre el tema se han concentrado en la población adulta, tanto en el caso de los ambientes campesinos o de la ciudad, como en el de las comunidades indígenas, no directamente en los niños.

$\mathrm{Al}$ comparar las estructuras conceptuales entre los dos grupos de participantes se destaca la diferencia entre los constructos de los que se valieron unos y otros; mientras que los primeros hicieron clasificaciones que corresponden a categorías de tipo científico, provenientes al parecer de los textos escolares, los segundos las establecen a partir de su experiencia directa.

Los niños indígenas no establecen diferencias entre animales domésticos y salvajes, y no se observaron diferencias en cuanto al número de animales temidos entre niños y niñas, como sí sucedió en el caso de los participantes de la sociedad mayoritaria colombiana. Los Uitoto diferencian claramente los animales que son de su territorio de los que no los son, hecho que no ocurrió con los participantes del primer grupo.

Los diferentes constructos y definiciones aportadas por los niños Uitoto se estructuran, principalmente, a partir de su participación activa en las jornadas sociales de la comunidad; desde muy pequeños son familiarizados con las actividades del hogar y con la participación en los eventos religiosos y ceremoniales que se realizan en la Maloca donde se congrega todos los días la comunidad.

Los aprendizajes inducidos por los adultos, para conocer el mundo en estos encuentros comunitarios, son reforzados con las experiencias obtenidas en sus acciones cotidianas; las faenas de cacería, las jornadas diarias de pesca y recolección de frutas y semillas, contribuyen a que los niños sean verdaderos conocedores de la selva. Sus juegos y prácticas recreativas en el río o en los árboles, determinan una cierta ventaja de conocimiento en relación con los niños "occidentales" o no indígenas. Gracias a estos mecanismos de aprendizaje es que los Uitoto logran estructurar elementos conceptuales para entender la naturaleza. 
Esta investigación realiza un aporte al campo de conocimiento de la educación y la pedagogía en lo que respecta a la importancia de formar a las nuevas generaciones en valores éticos, conocimiento ambiental y actitudes positivas hacia la naturaleza, y debe conducir a estudiar la relación humano-no humano de manera más directa, a través del conocimiento producto de las experiencias y tradiciones de las comunidades indígenas del país, que seguramente podrán enseñarnos cómo podemos organizar el conocimiento sobre la naturaleza gracias a las relaciones simbióticas que establecen con ella.

El aprendizaje sobre los animales en las escuelas y colegios rurales y de la ciudad parece estar divorciado de los encuentros directos con los animales en sus ambientes naturales, lo que produce poco entendimiento y pobres resultados en el conocimiento. Las enciclopedias, y libros "especializados" de los que se valen los maestros para enseñar conceptos sobre los animales incluyen datos, dibujos o fotografías, pero de forma poco interesante para los niños; estos materiales no educan realmente acerca de los animales reales en sus ambientes naturales.

Las oportunidades para exponer a los niños a la vida silvestre en los zoológicos son limitadas. En este sentido, Kellert (1980) ha mostrado que el conocimiento adquirido en los visitantes de un

\section{Bibliografía}

Alcock, J. (2001). The triumph of Sociobiology. New York: Oxford University Press.

Barraza, L., y Ceja, M. (2001). Los niños de la comunidad: su conocimiento ambiental y su percepción sobre la naturaleza. México: Universidad Autónoma de México.

Borg, I. y Groenen, P. J. F. (2005). Modern multidimensional scaling. New York: Springer.

Breakwell, G. M., y Canter, D. V. (1993). Empirical approaches to Social Representations. Oxford: Oxford university press.

Brown, S. (2002). 'Ethnic variations in pet attachment among students at an american school of veterinary medicine'. Society and animals, v. 10, no. 3, 249-266. zoológico no es diferente de forma significativa de quienes no lo han visitado, mientras que Morgan y Gramann (1989) creen que los zoológicos y los salones de clase son simplemente lugares donde los animales a menudo son simplemente expuestos a los espectadores.

Resulta importante que en los planes de estudio se haga énfasis en la visión ecológica, ética o moralista del valor de la naturaleza, en los recursos naturales de la región en la que se encuentre ubicada la escuela y el país, y en la importancia de su preservación y uso sostenible, soportada en experiencias directas como visitas a centros de educación ambiental o salidas de campo.

Sea que la interacción entre humanos y animales tenga o no vínculos arraigados en nuestra filogénesis, o sean más bien el producto de las construcciones sociales de la cultura a través de los discursos ambientalistas, como sostiene la teoría del aprendizaje y la postmodernidad, las categorías propuestas por Kellert para identificar las relaciones de las personas con la naturaleza representan un ordenador teórico, no inevitable, que permite integrar buena parte del sistema de constructos que establecen nuestros vínculos con la naturaleza, y que pueden orientar las investigaciones y la formación de actitudes hacia los animales.Д
Campos, C., Ulloa, A., y Rubio, H. (1996). Manejo de fauna con comunidades rurales. Bogotá: Fundación Natura.

Canter, D., Brown, J., y Groat, L. A. (1985). 'Multiple Sorting Procedure'. En: Brenner, M., Brown, J. y Canter, D. The Research Interview: Uses and Approaches, 79114. London: Academic Press.

Canosa, P., y Minguell, F. (2002). Niños y animales de compañía. Madrid: Editorial Debate.

Crawford, Ch. B., y Dennis L. K. (1998). Handbook of Evolutionary Psychology: ideas, issues, and applications. Mahwah, NJ: Lawrence Erlbaum.

De Civirieux, M. (1974). El hombre silvestre ante la naturaleza. Caracas: Monte Ávila Editores. 
Eisenberg, J. F. (1989). Mammals of the neotropics. The northern neotropics. Chicago y Londres: University of Chicago Press.

Emmons, L., y Feer, F. (1990). Neotropical rainforest mammals-A field guide-. Chicago y Londres: University of Chicago Press.

Feixas, G. (1995). 'Personal constructs in systemic practice'. En Neimeyer, R. A. y Mahoney, M. J. (Eds.), Constructivism in psychotherapy, 305-337. Washington, D. C.: American Psychological Association.

Feixas, G. (2003). 'Subjective methods'. En FernandezBallesteros, R. (Ed.) The enciclopedia of psychological assessment, v. II, 937-943. London: Sage.

Flynn, C. P. (2000). 'Battered women and their animal companions: symbolic interaction between human and nonhuman animals. Society and animals, v. 8, $\mathrm{n}^{\circ} .2,99-125$.

Fransella, F. (1996). George Kelly. Thousand Oaks, CA y Londres: Sage.

Galvis, C. y Tovar, H. (2007). Conceptualización de los niños indígenas Uitoto acerca de los animales. Tesis de Maestría. Bogotá: Universidad Pedagógica Nacional.

Kahan, P. H. (2001). The human relationship with nature: development and culture. Massachusetts: M.I.T Press.

Kellert, S. R. (1980). Phase II: Activities of the American Public Relating to Animals. United States: Department of the Interior Fish and Wildlife Service.

Kellert, S. R. (1997). Kinship to mastery: Biophilia in human evolution and development. Washington: Island Press.

Kellert, S. R. y Wilson, E. O. (1993). The Biophilia hypothesis. Washington D.C.: Island Press.

Kelly, G. A. (1955). The psychology of personal constructs. New York: Norton.

Kendall, H. y Lobao, L. (2004). Gender, place, and attitudes toward animals. Trabajo presentado en The annual meetings of the American Sociological Association, Agosto, San Francisco.

López, M. y Sueroke, L. (1993). Los animales de los Uitoto. Bogotá: Fundación Etnollano.
Mateos, A. (1998). 'Concepciones sobre algunas especies animales: ejemplificaciones del razonamiento por categorías. Dificultades de aprendizaje asociadas'. Revista Enseñanza de las Ciencias, 16 (1). Barcelona: Universidad Autónoma de Barcelona.

Morgan, J. M., y Gramann, J. H. (1989). 'Predicting effectiveness of wildlife education programs: A study of students' attitudes and knowledge toward snakes'. Wildlife Society Bulletin, 17 (4), 501-509.

Nassar, F. (2001). Actitud y pensamiento sobre la fauna silvestre en Colombia. Bogotá: Centro de Primatología Araguatos.

Ocampo, B. (2008). 'Análisis de contenido: un ejercicio explicativo'. En Páramo P. La investigación en ciencias sociales: Técnicas de recolección de información. Bogotá: Universidad Piloto de Colombia.

Páramo, P. (2008). La investigación en ciencias sociales: Técnicas de recolección de información. Bogotá: Universidad Piloto de Colombia.

Páramo, P., Arias, J. D., Melo, M. C., Pradilla, H., y Pabón, C. (1999). Nuestros vínculos con los animales. Bogotá: Universidad Pedagógica Nacional.

Peek, C. W., Dunham, C. C., y Dietz, B. E. (1997). ‘Gender, relational role orientation, and affinity foranimal rights'. Sex Roles, v. 37, Issue 11/12, 905-920.

Shepard, P. (1996). The others: how animals made us humans. Washington, D.C.: Island Press.

Strong, D. (1995). Crazy mountains: learning from wilderness to weightechnology. New York: State University of New York Press.

Townsend, W. (1989). 'Contribuciones a la etnozoología de la amazonía colombiana: el conocimiento zoológico entre los Huitoto'. Revista Colombia Amazónica. Bogotá.

Ulloa, A. (2002). ¿Ser humano? ¿Ser animal? Introducción'. Rostros culturales de la fauna. Las relaciones entre los humanos y los animales en el contexto colombiano. Bogotá: Instituto Colombiano de Antropología.

Ulrich, R. S. (1993). 'Biophilia, biophobia, and natural landscape. En Kellert, S. R. y Wilson, E. O. (Eds). The Biophilia hypothesis. Washington D. C: Island Press. 